\title{
Percutaneous management of peripheral vascular malformations: a single center experience
}

\author{
Barış Türkbey, Bora Peynircioğlu, Anıl Arat, Murat Canyiğit, Çiğdem Özer, İbrahim Vargel, Ferhun Balkancı, \\ Barbaros Çil
}

\begin{abstract}
PURPOSE
To review the therapeutic results of the combination of embolization and sclerotherapy, with or without surgery, in patients with peripheral vascular malformations (PVMs).

MATERIALS AND METHODS

A total of 40 patients ( 24 males and 16 females) with PVMs, who were treated via percutaneous embolization (transarterial [TA] versus direct puncture [DP]) and sclerotheraphy between March 2003 and September 2009, were included in this retrospective study. The mean age was 28 years (range, $6-66$ years), and 9 patients ( 7 boys, 2 girls) were $\leq 18$ years of age (range, 6-18 years). The 40 patients experienced 40 PVMs, of which 15 were localized to an upper extremity, 13 to a lower extremity, 7 to the axial body, and 5 to the pelvis. A total of 22 PVMs were high-flow, whereas 18 were low-flow. Indications for treatment included pain, swelling, extremity function loss, and cosmetic concerns.

RESULTS

A total of 85 embolization/sclerotheraphy sessions were performed ( 2.1 sessions per patient). For the 22 high-flow PVMs, 53 treatment sessions were completed (2.4 sessions per lesion). Of the high-flow PVMs, 10 were treated via embolization only (7 DP, 2 TA, 1 DP and TA), 5 via alcohol sclerotheraphy only (2 DP, 2 TA, 1 DP and TA) and 7 via a combination of embolization and sclerotheraphy ( $3 \mathrm{TA}, 4 \mathrm{DP}$ and TA). The agents of embolization and sclerotherapy were $n$-butyl cyanoacrylate $(n=22$ patients), Ony $x^{\oplus}(n=12$ patients), and alcohol ( $n=19$ patients). A total of 18 low-flow PVMs were treated in 32 sessions (1.8 sessions per lesion), all via the direct puncture approach. Of the low-flow PVMs, 11 were treated with embolization only, 6 with sclerotheraphy only, and 1 with a combined approach. In 16 patients (6 high-flow versus 10 low-flow), after a mean of 2.1 sessions (range, 1-9 sessions), the percutaneously treated lesions were excised by surgery without any major complications. In the 24 patients who did not have surgery, the lesions significantly decreased in size and the complaints from these patients improved. In four patients, skin ulcerations were identified, two of these patients needed surgical grafting; whereas in one patient, sciatic nerve paralysis developed after trans-arterial embolization and recovery was achieved in six months.

CONCLUSION

Percutaneous treatment of PVMs by embolization and sclerotheraphy is a safe and effective method, provided that appropriate lesion classification and treatment agent selection are performed.

Key words: • vascular malformation • embolization • sclerotherapy
\end{abstract}

From the Department of Radiology (B.T., B.P., M.C., Ç.Ö., F.B., B.C. $\$ barbaros@hacettepe.edu.tr), Hacettepe University School of Medicine, Ankara, Turkey; the Department of Radiology (A.A.), University of Wisconsin-Madison, Madison, Wisconsin, USA; and the Department of Plastic and Reconstructive Surgery (i.V.), Kırıkkale University School of Medicine, Kırıkkale, Turkey.

Received 21 August 2010; accepted 3 December 2010.

Published online 4 January 2011

DOI 10.4261/1305-3825.DIR.3808-10.0
P eripheral vascular malformations (PVMs) are among the most challenging lesions, both for diagnosis and for treatment. The vast majority of clinical findings are variable within a spectrum that ranges from no findings to life threatening findings, such as bleeding and congestive heart failure (1). Surgery has been the mainstay of standard treatment; however, endovascular treatments, including catheter-based percutaneous embolization and sclerotherapy with various agents, have recently produced favorable outcomes (2-13). In this study, the goal is to review the therapeutic results of various percutaneous techniques, including embolization and sclerotherapy in patients with PVMs.

\section{Materials and methods \\ Patient population and PVM lesions}

This retrospectively designed study was approved by the local institutional review board and informed consent was obtained from each adult participant and from the parents of the participants who were under 18 years of age. The study population included 40 consecutive patients (24 males and 16 females) with a mean age of 28 years (range, 6-66 years) who had a total of 40 PVMs and underwent elective percutaneous embolization or sclerotherapy for the treatment of PVM between March 2003 and September 2009. Of this subject group, 9 patients (7 boys, 2 girls) were $\leq 18$ years of age (ranging from 6 to 18 years). All patients were referred to Interventional Radiology by the Plastic and Reconstructive Surgery Department for the presence of at least one of the following complaints: pain, swelling, extremity function loss, and cosmetic concerns. The method of treatment was made on a patient-by-patient basis by a multidisciplinary consensus that included plastic surgeons and interventional radiologists. The locations of the PVMs in the subject group included the upper extremity $(n=15)$, lower extremity $(n=13)$, axial body $(n=7)$, and pelvis $(\mathrm{n}=5)$. All patients underwent a detailed physical examination and all PVM lesions were evaluated by color Doppler ultrasound, magnetic resonance imaging (MRI), or catheter angiography (only for the high-flow vascular malformations) for classification according to their flow patterns (low versus high), which has been defined in detail elsewhere (2).

\section{Percutaneous management techniques: embolization and sclerotherapy procedures}

Embolization is the intentional occlusion of the nidus and feeder vessels of a PVM via a foreign material (e.g., n-butyl cyanoacrylate (NBCA) [Histoacryl, Braun Medical, Melsungen, Germany] or ethylene-vinyl alcohol copolymer $\left(\right.$ Onyx $\left.{ }^{\circledR}\right)$ [Micro Therapeutics, Irvine, California, USA], [Table 1]); whereas sclerotherapy is the obliteration of a PVM via an aggressive sclerosing agent (e.g., alcohol [Table 1]) that causes instant precipitation of the endothelial cell proteins through denaturation and 
cellular dehydration and ultimately results in endothelial destruction and thrombosis (3). In the current study, all of the embolization and sclerotherapy procedures were performed in an interventional radiology unit under general anesthesia. Transarterial (TA) catheterization and/or direct puncture (DP) techniques were chosen, depending on the flow characteristics of the PVM lesion. The TA catheterization approach was preferred for high-flow PVMs, whereas the DP approach was generally used for low-flow PVMs. However, even in high-flow lesions, DP with or without distal compression was needed in a portion of the cases to completely embolize the nidus itself. For TA embolization in high-flow PVMs, the most proximal main feeding artery of the PVM was first catheterized using a $4 \mathrm{~F}$ diagnostic catheter through a 4-5 $\mathrm{F}$ arterial sheath placed in the right femoral artery, followed by a selective arteriogram. Then, the distal feeder arteries were super-selectively catheterized with a microcatheter using a co-axial technique. Eventually, the desired embolization or sclerotherapy agents (Table 1 ) were injected under the guidance of fluoroscopy. Both the decision to perform sclerotherapy or embolization and the choice of embolic agent was primarily made at this phase of the procedure and depended on the volume of the PVM and how close the tip of the microcatheter could be placed to the actual nidus. Of course, one of the most important factors for choosing the agent used for sclerotherapy/embolization was the presence (or lack) of a surgical plan and the timing of the surgery. After embolization and sclerotherapy procedures, control angiograms were performed. Hemostasis was achieved by manual compression following removal of the catheters, the guidewire and the arterial sheath. In the DP technique for high-flow PVM, a 19-23 G butterfly needle(s) or $21 \mathrm{G}$ micro-puncture needle(s) was placed into the nidus or into the closest arterial or venous site to the PVM and embolization agents (Table 1) were injected under the guidance of fluoroscopy; in the meantime, to guarantee the complete obliteration of the nidus, distal compression was performed when possible. In the DP technique for low-flow PVM, following the appropriate preparation and draping of the puncture site, again a 19-23 G but-

Table 1. Embolization and sclerotherapy agents

\begin{tabular}{llccc}
\hline & & & \multicolumn{2}{c}{ Cases examined } \\
\cline { 4 - 5 } Agent & Information & Delivery route & High-flow & Low-flow \\
\hline NBCA $^{\text {a }}$ & $\begin{array}{l}\text { Histoacryl, Braun Medical, } \\
\text { Melsungen, Germany }\end{array}$ & TA, DP & $10 / 22$ & $12 / 18$ \\
Onyx & $\begin{array}{l}\text { Micro Therapeutics, Irvine, } \\
\text { California, USA }\end{array}$ & TA, DP & $11 / 22$ & $1 / 18$ \\
Ethanol & NA & TA, DP & $12 / 22$ & $7 / 18$ \\
\hline
\end{tabular}

NBCA, n-butyl cyanoacrylate; TA, transarterial; DP, direct puncture; NA, not applicable aNBCA was diluted to a ratio of $1 / 6$ with iodized oil (Lipiodol, Laboratorie Guerbet, Roissy, France). ${ }^{b}$ Catheters were flushed with di-methyl sulfoxide (DMSO) prior to ethylene-vinyl alcohol copolymer (Onyx) injections.

Table 2. Classification of peripheral vascular malformations (PVM) according to their location and flow pattern

\begin{tabular}{llccc}
\hline & & \multicolumn{2}{c}{ PVM flow characteristics } & \\
\cline { 3 - 4 } & & High-flow & Low-flow & Total \\
\hline PVM location & Upper extremity & 8 & 7 & 15 \\
& Lower extremity & 8 & 5 & 13 \\
& Pelvis & 5 & 0 & 5 \\
Total & 1 & 6 & 7 \\
& Axial body & 22 & 18 & 40
\end{tabular}

terfly needle(s) or $21 \mathrm{G}$ micro-puncture needle(s) was placed into the PVM (mostly under ultrasound guidance), diluted contrast was injected into the PVM to depict the vascularity and venous drainage of the PVM and embolization or sclerotherapy agents (Table 1) were injected under the guidance of fluoroscopy. Additional punctures were performed when needed during the DP technique. In both techniques (TA and DP), complete obliteration of the PVM was the goal; for diffuse, large PVMs, additional treatment sessions were performed at 4-6 weeks intervals. Because the dose limitations are important for some agents, such as alcohol and lipiodol, session plans were generally made beforehand, which also affected the surgical plan. Antibiotic prophylaxis and steroid medications were used 3-5 days before and after the treatment sessions in most of the patients, depending on the location and the size of the PVM. Periodic (1-3 months) follow-up evaluations were performed based on the physical examination, using gray scale and/or color Doppler ultrasound and MRI as needed. In the current study, technical success in the embolization and sclerotherapy procedures was defined as a successful catheterization and a subsequent partial or complete obliteration of the PVM lesion via embolization or sclerotherapy; clinical success was defined as an improvement in the initial symptoms of the patient following percutaneous intervention in the non-surgery group and resection of the percutaneously treated PVM without major intraoperative bleeding.

\section{Results}

Pre-procedural imaging studies demonstrated 22 high-flow and 18 low-flow PVMs in 40 patients. Classification of the PVMs according to their location and flow pattern is summarized in Table 2. The 40 PVM in 40 patients were treated with a total of 85 sessions (2.1 sessions per patient). A total of 22 high and 18 low-flow PVM lesions were treated in 53 ( 2.4 sessions per lesion) and 32 (1.8 sessions per lesion) sessions, respectively. Of the high-flow PVM lesions, 10 were treated with embolization only, five with sclerotherapy only, and seven with combined embolization and sclerotherapy; whereas 11 low-flow PVM lesions were treated with embolization only, six with scle- 
Table 3. Treatment methods used in peripheral vascular malformations (PVM)

\begin{tabular}{llccc}
\hline & & \multicolumn{2}{c}{ PVM flow characteristics } & \\
\cline { 3 - 4 } & & High-flow & Low-flow & Total \\
\hline $\begin{array}{l}\text { Treatment } \\
\text { method }\end{array}$ & Embolization & $10(2.3 / 1-6)$ & $11(1.27 / 1-3)$ & $21(1.76 / 1-6)$ \\
& Sclerotherapy & $5(2 / 1-4)$ & $6(1.5 / 1-2)$ & $11(1.7 / 1-4)$ \\
& $\begin{array}{l}\text { Combined embolization } \\
\text { and sclerotherapy }\end{array}$ & $7(2.85 / 1-5)$ & $1(9 / \mathrm{NA})$ & $8(3.6 / 1-9)$ \\
Total & $22(2.4 / 1-6)$ & $18(1.8 / 1-9)$ & $40(2.15 / 1-9)$
\end{tabular}

Numbers in parentheses represent the mean treatment sessions/range of number of treatment sessions. $\mathrm{NA}$, not applicable

Table 4. Treatment approach routes used in peripheral vascular malformations (PVM)

\begin{tabular}{llccc}
\hline & & \multicolumn{2}{c}{ PVM flow characteristics } & \\
\cline { 3 - 4 } & & High-flow & Low-flow & Total \\
\hline $\begin{array}{l}\text { Treatment } \\
\text { approach } \\
\text { route }\end{array}$ & Transarterial & $7(2.85 / 1-6)$ & $0(\mathrm{NA})$ & $7(2.85 / 1-6)$ \\
& Direct puncture & $9(1.33 / 1-2)$ & $18(1.8 / 1-9)$ & $27(1.63 / 1-9)$ \\
& $\begin{array}{l}\text { Transarterial and } \\
\text { direct puncture }\end{array}$ & $6(3.5 / 1-6)$ & $0(\mathrm{NA})$ & $6(3.5 / 1-6)$ \\
Total & $22(2.4 / 1-6)$ & $18(1.8 / 1-9)$ & $40(2.15 / 1-9)$ \\
\hline
\end{tabular}

Numbers in parentheses represent the mean treatment sessions/range of number of treatment sessions. NA, not applicable

rotherapy only and one with combined embolization and sclerotherapy (Table 3). A total of seven high-flow PVM lesions were treated using the TA approach, nine with DP and six with a combination of TA and DP; whereas all low-flow PVM lesions were treated with the DP approach (Table 4). All of the percutaneous treatment procedures were successful, and non-targeted embolization or sclerotherapy was not encountered (Figs. 1 and 2).
In the follow up, for the 24 patients (16 high-flow, 8 low-flow PVMs) in whom surgical resection was not planned and performed, the PVM lesions diminished in size and the admission complaints improved after a mean of 2.1 percutaneous treatment sessions (range, 1-6 sessions) (Table 5). In sixteen patients (6 high-flow, 10 low-flow), who had a mean percutaneous treatment session number of 2.1 (range, 1-9 sessions), surgical resection was performed in 7 days after completion of the embolization and sclerotherapy session(s), as planned prior to percutaneous treatment, and no major complication (e.g., bleeding) was observed during surgery (Table 5).

Overall, for the embolization and sclerotherapy procedures, the technical success rate was $100 \%$, whereas the clinical success rate (improvement in any of the initial symptoms: pain, loss of function, cosmetic concern or a successful/ uneventful eventual surgical resection/ curettage) was $100 \%$ in both the surgery and the non-surgery groups. As for complications, skin ulceration was observed in four patients who underwent sclerotherapy without subsequent surgical resection, and two of these patients underwent successful surgery for these skin ulcerations, whereas two recovered without any requirement for intervention. One patient, who had combined
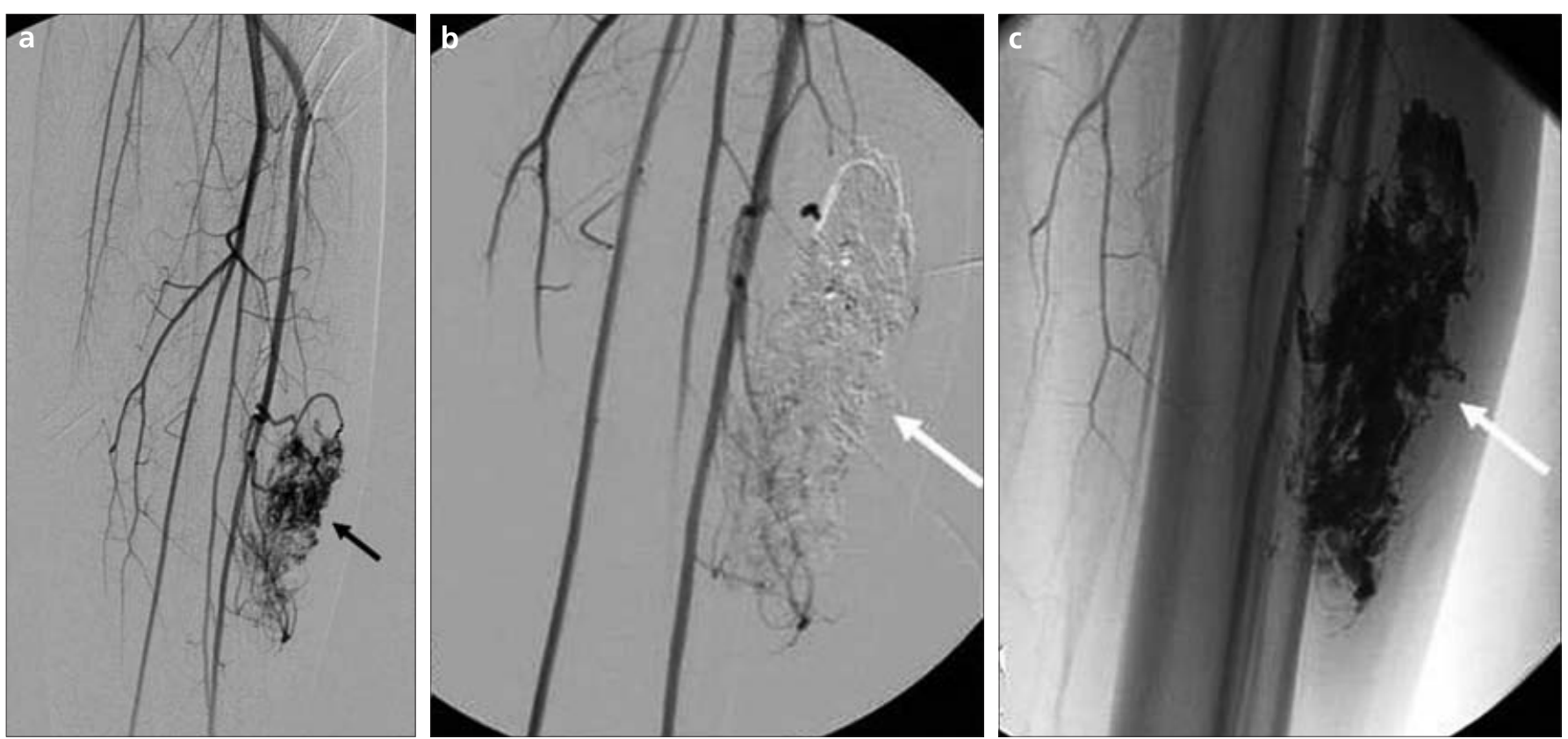

Figure 1. a-c. A 20-year-old woman with a left lower extremity high-flow vascular malformation. The diagnostic angiogram (a) demonstrates a high-flow vascular malformation mainly fed by the left anterior tibial artery (black arrow). Control digital subtraction (b) and inverted native (c) angiograms after NBCA embolization by the direct puncture route demonstrate complete obliteration of the vascular malformation lesion (white arrows). The patient underwent a surgical excision without complication. 

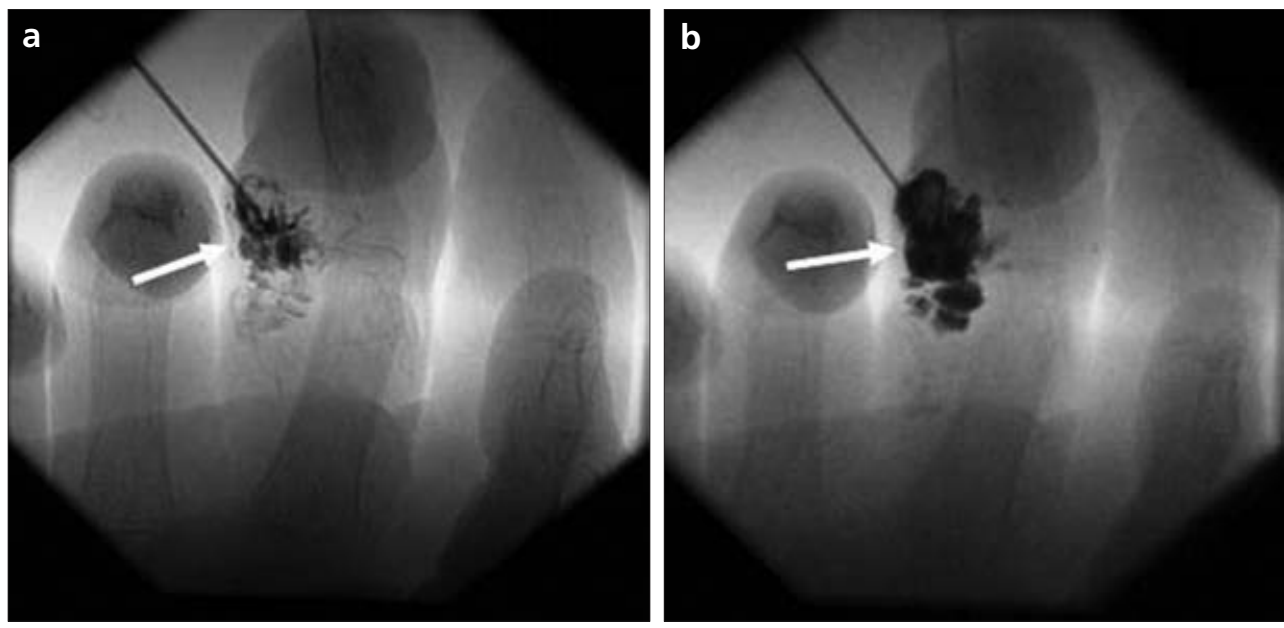

Figure 2. a-d. A 9-year-old boy with a left middle finger low-flow vascular malformation. Serial fluoroscopy images (a-d) demonstrate NBCA embolization of the vascular malformation lesion in two sessions (arrows). The patient had surgical excision without complication.
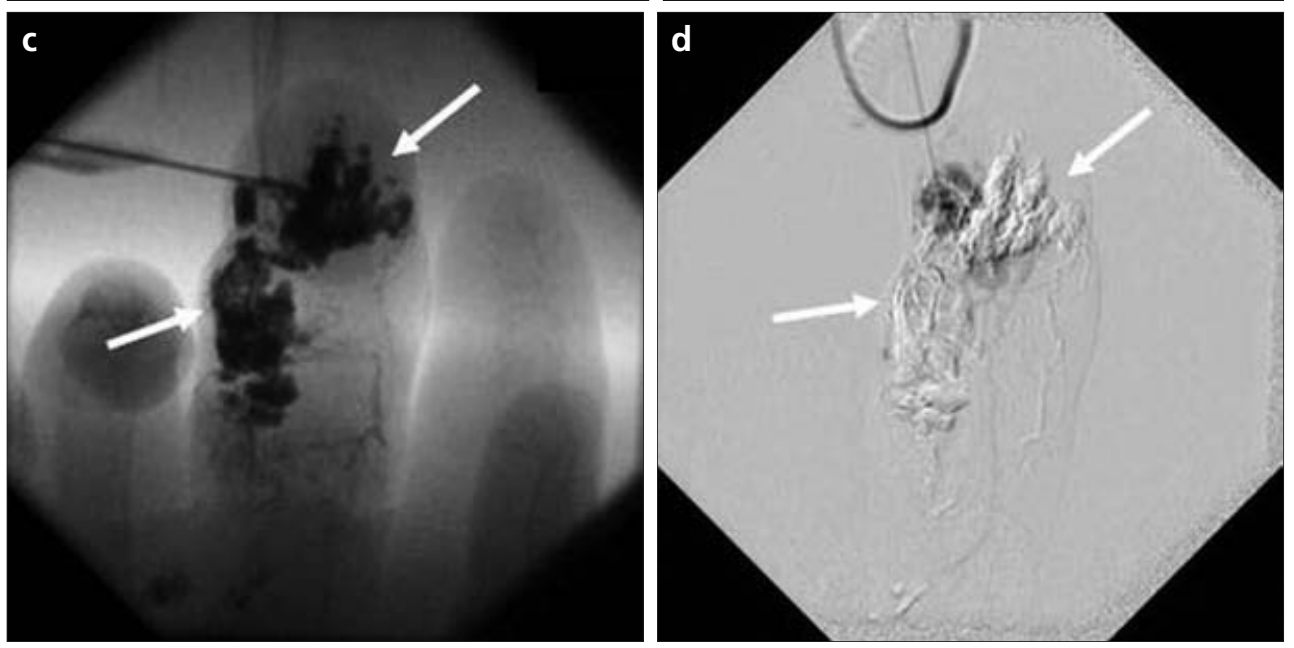

embolization and sclerotherapy for a high-flow pelvic PVM, developed sciatic nerve paralysis after the sclerotherapy session, but significant improvement was observed in the paralysis findings in the first six month interval.

\section{Discussion}

The management of PVM lesions is challenging due to their complexity and because of the difficultly inherent in selecting the appropriate treatment approach. Surgical excision, which includes excision of the nidus and liga-

Table 5. Course after percutaneous treatment of peripheral vascular malformations (PVM)

\begin{tabular}{|c|c|c|c|c|}
\hline & & \multicolumn{2}{|c|}{ PVM flow characteristics } & \multirow[b]{2}{*}{ Total } \\
\hline & & High-flow & Low-flow & \\
\hline \multirow{2}{*}{$\begin{array}{l}\text { Course after } \\
\text { percutaneous } \\
\text { treatment }\end{array}$} & $\begin{array}{l}\text { Improvement with } \\
\text { surgical resection }\end{array}$ & $6(2.16 / 1-6)$ & $10(2.1 / 1-9)$ & $16(2.12 / 1-9)$ \\
\hline & $\begin{array}{l}\text { Improvement without } \\
\text { surgical resection }\end{array}$ & $16(2.5 / 1-6)$ & $8(1.37 / 1-2)$ & $24(2.12 / 1-6)$ \\
\hline Total & & $22(2.4 / 1-6)$ & $18(1.8 / 1-9)$ & $40(2.15 / 1-9)$ \\
\hline
\end{tabular}

Numbers in parentheses represent the mean treatment sessions/range of number of treatment sessions. tion of the feeding vessels, offers an attractive solution, in theory. However, the infiltrating nature of PVMs, specifically those that are high-flow and large, increases the possibility of recurrence and complications; moreover, a failure during surgical excision can result in the loss of an endovascular treatment attempt. The combination of embolization and sclerotherapy can potentially serve as a treatment method. The utility of percutaneous treatments with various agents, including NBCA, polyvinyl alcohol particles (PVA), Onyx and etha- nol, has been reported (6-27). Rockman et al. (6) treated 50 patients with PVMs and used either TA NBCA or DP ethanol to treat high- or low-flow PVMs, respectively; $92 \%$ of their patients recovered, whereas $6 \%$ needed additional surgical intervention and one patient lost an extremity. Lee et al. (7) reported on 76 patients with PVMs, of whom, 48 ( $n=16$, post embolization/sclerotherapy surgery; $n=25$, total recovery; $n=7$, partial recovery) received embolization/sclerotherapy with NBCA, coils and ethanol. Osuga et al. (8) reported on the embolization of PVMs with PVA in 20 patients and discussed the safety and feasibility of embolization via PVA. Tan et al. (18) proposed the requirement for multiple session embolization of high-flow PVMs in 13 patients who were treated with NBCA and PVA. Donnelly et al. (9) reported successful sclerotherapy under the guidance of ultrasound and fluoroscopy in 19 patients with lowflow vascular malformations. Górriz et al. (11) reported complete recovery in 14 patients with intramuscular low- 
flow PVM after ethanol sclerotherapy. Castenada et al. (16) and Numan et al. (17) reported on the use of Onyx embolization for low- and high-flow PVMs with various degrees of recovery in two and nine patients, respectively.

In the current study, the embolization and sclerotherapy was technically successful in $100 \%$ of the patients. For the majority of the high-flow PVM lesions, the TA approach was the preferred technique, whereas DP was mainly used as an adjunct to ensure complete obliteration of the lesion. Surgical excision was performed in six patients, all of whom had embolization with NBCA and/or Onyx. Symptomatic relief was achieved in all patients who did not undergo surgery and the complication rate for the treatment of high-flow PVM lesions was $22.7 \%(5 / 22 ; n=4$ skin ulceration, $\mathrm{n}=1$ transient sciatic nerve paralysis); however, all of the patients recovered from their complications without permanent damage or mortality. For all of the lowflow PVM lesions, the DP approach was preferred and adjunct surgical excision was performed in 10 patients, all of whom had embolization with NBCA, whereas symptomatic relief was achieved in the remaining 8 patients. No complication or procedure-related mortality was encountered. Our results for the management of PVM lesions and the complication rates were comparable with other studies in the literature $(18,19,21,24)$.

Currently, there is no consensus on the selection of an appropriate agent for endovascular treatment. The flow pattern of the lesion, the location (deep vs. superficial), operator experience, the presence of an adjunct surgery plan, cost, and availability are the main factors that influence agent selection. The selection of an agent was based on these criteria in the current study. We usually preferred ethanol sclerotherapy or NBCA embolization via the DP route for superficial low-flow PVM lesions, TA embolization with NBCA and/or Onyx was used for deeply located high-flow lesions and NBCA embolization was preferred in patients with an adjunct surgery plan to increase the success rate of excision.

In conclusion, PVM lesions are rare and present challenges in both diagnosis and management. Percutaneous management with embolization and sclerotherapy can be effectively used alone or with surgery for the treatment of PVM lesions, provided that the lesion is correctly classified and an appropriate agent is selected. To achieve better outcomes for these potentially complex lesions, interventional radiologists and plastic surgeons must work together, beginning with the diagnosis and continuing throughout the treatments, so that these lesions can be treated aggressively and patiently, yielding excellent outcomes with an acceptable rate of complications. The choices of the embolization/sclerotherapy route and of the embolic agent play an important role in the management of these lesions and require significant experience and expertise in all kinds of imaging-guided embolization.

\section{Conflict of interest disclosure}

The authors declared no conflicts of interest.

\section{References}

1. Rutherford RB. Congenital vascular malformations: diagnostic evaluation. Semin Vasc Surg 1993; 6:225-232.

2. Jackson IT, Carreno R, Potparic Z, Hussain K. Hemangiomas, vascular malformations, and lymphovenous malformations: classification and methods of treatment. Plast Reconstr Surg 1993; 91:1216-1230.

3. Rosenblatt M. Endovascular management of venous malformations. Phlebology 2007; 22:264-275.

4. Olcott C, Newton TH, Stoney RJ, Ehrendeld WK. Proceedings: management of arteriovenous malformations. J Cardiovasc Surg (Torino) 1976; 17:101.

5. Flye MW, Jordan BP, Schwartz MZ. Management of congenital arteriovenous malformations. Surgery 1983; 94:740-747.

6. Rockman CB, Rosen RJ, Jacobowitz GR, et al. Transcatheter embolization of extremity vascular malformations: the long-term success of multiple interventions. Ann Vasc Surg 2003; 17:417-423.

7. Lee BB, Do YS, Yakes W, Kim DI, Mattassi $\mathrm{R}$, Hyon WS. Management of arteriovenous malformations: a multidisciplinary approach. J Vasc Surg 2004; 39:590-600.

8. Osuga K, Hori S, Kitayoshi $\mathrm{H}$, et al. Embolization of high flow arteriovenous malformations: experience with use of superabsorbent polymer microspheres. J Vasc Interv Radiol 2002; 13:1125-1233.

9. Donnelly LF, Bissett GS 3rd, Adams DM. Combined sonographic and fluoroscopic guidance: a modified technique for percutaneous sclerosis of low-flow vascular malformations. AJR Am J Roentgenol 1999; 173:655-657.

10. Dubois J, Garel L. Imaging and therapeutic approach of hemangiomas and vascular malformations in the pediatric age group. Pediatr Radiol 1999; 29:879-893.

11. Górriz E, Carreira JM, Reyes R, et al. Intramuscular low flow vascular malformations: treatment by means of direct percutaneous embolization. Eur J Radiol 1998; 27:161-165.
12. Kadono T, Kishi A, Onishi Y, Ohara K. Acquired digital arteriovenous malformation: a report of six cases. Br J Dermatol 2000; 142:362-365.

13. Kazekawa K, Kawano T, Kawaguchi T, et al. Newly synthesized liquid embolization material for arteriovenous malformation. J Clin Neurosci 1998; 5:45-48.

14. Yakes WF, Haas DK, Parker SH, et al. Symptomatic vascular malformations: ethanol embolotherapy. Radiology 1989; 170:1059-1066.

15. Shireman PK, McCarthy WJ, Yao JS, Vogelzang RL. Treatment of venous malformations by direct injection with ethanol. J Vasc Surg 1997; 26:838-844.

16. Castaneda F, Goodwin SC, Swischuk JL, et al. Treatment of pelvic arteriovenous malformations with ethylene vinyl alcohol copolymer (Onyx). J Vasc Interv Radiol 2002; 13:513-516.

17. Numan F, Omeroglu A, Kara B, Cantaşdemir M, Adaletli I, Kantarci F. Embolization of peripheral vascular malformations with ethylene vinyl alcohol copolymer (Onyx). J Vasc Interv Radiol 2004; 15:939-946.

18. Tan KT, Simons ME, Rajan DK, Terbrugge K. Peripheral high-flow arteriovenous vascular malformations: a single-center experience. J Vasc Interv Radiol 2004; 15:10711080.

19. Do YS, Yakes WF, Shin SW, et al. Ethanol embolization of arteriovenous malformations: interim results. Radiology 2005; 235:674-682.

20. Bae S, Do YS, Shin SW, et al. Ethanol embolotherapy of pelvic arteriovenous malformations: an initial experience. Korean J Radiol 2008; 9:148-154.

21. Cho SK, Do YS, Kim DI, et al. Peripheral arteriovenous malformations with a dominant outflow vein: results of ethanol embolization. Korean J Radiol 2008; 9:258267.

22. White RI Jr, Pollak J, Persing J, Henderson $\mathrm{KJ}$, Thomson JG, Burdge CM. Long-term outcome of embolotherapy and surgery for high-flow extremity arteriovenous malformations. J Vasc Interv Radiol 2000; 11:1285-1295.

23. Suh JS, Shin KH, Na JB, Won JY, Hahn SB. Venous malformations: sclerotherapy with a mixture of ethanol and lipiodol. Cardiovasc Intervent Radiol 1997; 20:268273.

24. Sofocleous CT, Rosen RJ, Raskin K, Fioole B, Hofstee DJ. Congenital vascular malformations in the hand and forearm. J Endovasc Ther 2001; 8:484-494.

25. Arat A, Cil BE, Vargel I, et al. Embolization of high-flow craniofacial vascular malformations with onyx. AJNR 2007; 28:14091414.

26. Cil BE, Vargel I, Geyik S, Peynircioglu B, Cavusoglu T. Venous vascular malformations of the craniofacial region: pre-operative embolisation with direct percutaneous puncture and N-butyl cyanoacrylate. Br J Radiol 2008; 81:935-939.

27. Konas E, Canter HI, Cil B, et al. Volumetric assessment of results of treatment of vascular malformations of the head and neck regions treated with a minimally invasive surgical technique after embolization procedure. J Craniofac Surg 2009; 20:402-405. 